Research Article

\title{
The Physical Health Evaluation of Adolescent Students Based on Big Data
}

\author{
Chunyan Fan \\ Taiyuan University of Technology, Taiyuan 030024, China \\ Correspondence should be addressed to Chunyan Fan; fanchunyan@tyut.edu.cn
}

Received 12 October 2021; Revised 6 December 2021; Accepted 9 December 2021; Published 22 December 2021

Academic Editor: Ateeq Rehman

Copyright (c) 2021 Chunyan Fan. This is an open access article distributed under the Creative Commons Attribution License, which permits unrestricted use, distribution, and reproduction in any medium, provided the original work is properly cited.

Globally, it is widely accepted that the physical health of the young generation is continuously declining which remained unnoticed in many countries including China. Therefore, it is very important to scientifically investigate the evaluation index system and effectively interrogate the current health position of the young generation especially students. The results regarding health issues will be further investigated in terms of finding the optimal solutions for improving the status quo. Considering adolescent physical health evaluation indicators of Chinese students, this paper introduces the new era of adolescent physical health evaluation which should focus on "health quality indicators" for analyzing the objectivity and applicability of the indicators through testing and research methods. The method of simulation experiment research is used to demonstrate the scientificity and validity of the index. The optimization of the physical health evaluation indicators of adolescent students helps in assessing the health quality of adolescents, scientifically and accurately. In addition, it can effectively improve the physical health problems faced by adolescents. The proposed model achieves the evaluation indicators of adolescent health quality, collects physical health information and exercise data of adolescent students in a certain area of the northwest through big data, and conducts research as the research object. Furthermore, through the collation and analysis of the correlation data of the aerobic capacity evaluation indicators, the results show that the mid-run test value currently used in China is negatively correlated with the relative value of the measured maximum oxygen uptake on the treadmill.

\section{Introduction}

The healthy development of the physique of adolescents in China has gone through a relatively long history. It has experienced different historical development periods. In the development process of different times, a series of evaluation standards for testing and evaluating the physical health of adolescent students have been formulated and promulgated, such as "Labor and Health System," "National Student Physical Health Standard," and "Student Physical Health Standard." The formulation of these standards conformed to the development level of society, economy, culture, and education at that time and also achieved the improvement of the physical health of young people and the cultivation of allround development talents [1-3]. The various indicators in the standard objectively and effectively evaluate the basic conditions of adolescents' physical development, physical function, and physical fitness.
With the passage of time and advancement in technology, people's understanding of health is constantly improving. Currently, care for life and pursuing health conditions are the main goals of our society. Therefore, facing the outstanding physical health problems that have emerged in the youth group in recent years, a scientific evaluation system has been established to stimulate the health consciousness of young students, for instance, changing bad lifestyles, developing good habits of long-term exercise, and effectively promoting the healthy development of young students' physique [4]. In recent years, schools at all levels across the country have continuously promoted health education and health promotion. For example, in 2011, Shanghai launched the health education promotion project. Through the popularization and guidance of physical education and extracurricular health education, it effectively interferes with the behavior of young students and promotes health levels $[5,6]$. As an evaluation system, our country's 
student physical health standard is to monitor the coordination between the health of adolescents and the implementation of physical activities and physical education courses. It is also an evaluation to better promote the reform of physical education contents and teaching methods and play a positive guiding role. Countries around the world have generally pointed out in the adolescent physical health monitoring report that the aerobic endurance level of adolescents has a continuous decline in the adverse development trend [7].

Researchers also pointed out that the current aerobic capacity and cardiopulmonary endurance levels of adolescents in China have declined severely. Aerobic work capacity, as an important indicator of adolescent physical health evaluation, has always been concerned and studied by scholars from all over the world [8]. Good aerobic work ability is useful for reducing the risk of cardiovascular and cerebrovascular diseases, reducing the systolic and diastolic blood pressure in the resting state, reducing individual fat content and intra-abdominal fat content, and reducing the incidence of central obesity. At the same time, it also helps to reduce anxiety, and relieve stress, increase happiness, and enhance daily work ability and athletic ability. At present, the middle running ( $1000 \mathrm{~m}$ for boys and $800 \mathrm{~m}$ for girls) and step test are selected as the evaluation standards for the physical health of adolescents in China. Foreign countries use the 20-meter shuttle run test level 7 (20-mSRT) for evaluating aerobic capacity. At present, the effectiveness and reliability of the 20-meter return run test have been relatively consistent at home and abroad. The consensus has also been adopted by many foreign countries in the evaluation of adolescent physical health [9]. Maximum oxygen uptake $\left(\mathrm{VO}_{\max }\right)$ is the most convincing indicator to assess the human cardiopulmonary function and aerobic endurance capacity, reflecting the human body's oxygen transport system and muscle transport, uptake, and utilization of oxygen [10]. It is usually used to evaluate the body's cardiovascular function in exercise physiology research. Studies have shown that maximum oxygen consumption is closely related to the performance of endurance events which is selected by domestic and foreign researchers as the "gold standard" for evaluating aerobic capacity [11].

In the light of the above literature and problems therein, this paper focused on investigating various evaluation indicators to maximize oxygen uptake. It is evident from the results that through the collation and analysis of the correlation data of the aerobic capacity evaluation indicators, the mid-run test value currently used in China is negatively correlated with the relative value of the measured maximum oxygen uptake on the treadmill. Hence, in the middle running project, anaerobic exercise is the main energy supply mode. In aerobic and anaerobic mixed exercise, more than half of the energy during the test is provided by the anaerobic system Furthermore, oxygen uptake is also evaluated for both groups by a $20 \mathrm{~m}$ run test. The experimental results show that it is more appropriate to select the $20 \mathrm{~m}$ reentry run as a test index to evaluate the aerobic capacity of adolescents.
In Section 2, related work of this contribution is presented while in Section 3, the proposed research methodology is illustrated with details of parameter and test selection. In Section 4, the results achieved from evaluating different tests and parameters are discussed in the light of existing studies. Finally, the paper is concluded in Section 5.

\section{Related Work}

In different periods of development, China has formulated a series of systems and standards in order to promote the healthy development of the national physique and enhance the physical health of young students. Reviewing the physical health evaluation standards of adolescent students in different periods since the founding of the People's Republic of China will help us to understand the trend of the health development of adolescents in the country more clearly. At the same time, it will also help to formulate an evaluation system that is more in line with the health development of adolescents in the country. During the period from 1951 to 1963 , when China fully implemented the "Labor and Health System," due to the recovery of the national economy, poor school sanitation and malnutrition affected the development of students' physical health, and the overall level was at a low level. However, from 1975 to 2002, and during the implementation of the "National Physical Exercise Standards," the rapid growth of the national economy, the rapid development of scientific and technological information, the increase of static lifestyles, the reduction of physical activity, the breeding of "civilization diseases," and other factors led to a decline in the physical health of adolescents [12]. The "Student Physical Health Standard (Trial Plan)" was implemented in 2002. While modern civilization has brought rich material of enjoyment which also brought new threats to health. More young students suffer from insufficient exercise, overnutrition, and at the same time, other influencing factors such as environmental pollution. The physical health of teenagers and children in the new era is also facing challenges [13]. In 2007, the Ministry of Education and the State Sports General Administration revised and improved the "Students' Physical Health Standards (Trial Plan)" on the basis of conscientiously summarizing the pilot work in various regions, and named them the "National Student Physical Health Standards" as the "National Student Physical Exercises." The organic part of the standard is fully implemented in domestic schools [14, 15]. The "National Student Physical Fitness and Health Standard" highlights the evaluation concept based on health quality and the implementation effect of one standard with multiple uses and sets various test indicators for sports technical ability. Its purpose is to improve the youth's performance through sports technical project exercise physical fitness level. The results of the fifth national student physical health survey in 2005 show that on the whole, while the level of physical development continues to improve, physical fitness, muscle strength, and muscle endurance are still showing a further downward trend, and the detection rate of obesity and poor eyesight continues to rise, and the overall situation is not optimistic. 
The sixth national survey on student physique and health began in 2010, and the results showed that the physical and health status of young students has improved overall, and the decline in the physical quality of young students has begun to be controlled. The detection rate of obesity and poor eyesight is still increasing. Judging from the six surveys and monitoring of the physical health of adolescent students in China from the last 25 years, we have accumulated detailed data on the physical health of adolescents at different ages, reflecting the trend of the healthy development of adolescents in China and laying a solid foundation for promoting the healthy development of adolescents [16].

Countries all over the world attach great importance to the research and practice of physical fitness testing projects. The international committee for physical fitness research (CPFR) announced various physical fitness testing implementation plans in 1974 [17]. The four aspects of indicators, morphometry, body composition, and basic physical fitness testing are recognized by the International Biological Development Planning Commission (IBP). They believe physical fitness testing should include growth and morphological development, genetic function, functional capacity, and lung function [18]. Applicability to climate, evaluation of nutritional status, medical and metabolic research, sociological evaluation of population, and analysis of relevant social and cultural factors summarize the healthy development of foreign adolescents as follows. Since the 1980s, the United States has developed and launched a series of school-based youth physical health education programs, such as "SPARK" and "CATCH" related to physical education courses and physical education programs; "FITNESS FOR LIFE," a learning program related to the concept and connotation of health; and physical testing such as the health quality monitoring system "FITNESSGRAM," "PHYS CAL BEST," and so on. The implementation of these health promotion plans has strongly promoted the life-long, personalized, and fitness-oriented reform of physical education in American schools, and also promoted the focus of school physical education is shifted from "student physical education" to "student fitness education" [19]. Although each state in the United States has its own physical fitness test program, it is mainly based on the President's challenge and the FITNESSGRAM test system. These two test standards have been implemented for a long time in the physical test of young students in the United States.

On the other hand, Japan has the most comprehensive data on the physical fitness of adolescents and children. It has accumulated all the data on the growth and development of adolescents for more than 100 years, since 1898. The Meiji government of Japan issued the "Notice on Physical Examination of Adolescents" emphasizes the importance of the health problems of adolescents. The Japanese research on the evaluation of adolescent physical health tests is called physical research [20]. In 1879, Japan began the work of physical testing of adolescents, which was tested separately. Japanese scholars divide physical strength into two categories: action physical strength and defensive physical strength. The test indicators have always been revised continuously with the reform and development of school physical education and the change of national sports concepts.
The European Union member states adopt a relatively uniform method. Test standards are used to determine the physical health of young people, which is conducive to the comparison of the physical health of young people in different countries and the comparison of physical education in various countries, so as to better carry out the physical health of nationals in various countries. In 1978, the relevant adolescent physical health test agreement was signed. After years of research and exploration, the test standard guide and physical fitness test content were published in 1986.

The Russian government is also very concerned about the development of adolescent physical health. In the 21st century, the Russian government has the current physical and healthy development of young students; it is required that the government increases the investment and construction in sports and create good resources for mass fitness activities for the people and pay attention to the physical development of young people. In 2009, the "Sports Development Strategy of the Russian Federation to 2020" emphasizes the creation of good sports facilities for students, encourages students to exercise, and develop a healthy lifestyle.

\section{Method}

3.1. Research Object. In this study, a total of 90 students were selected based on the research data collected by big data, and the basic information of the subjects (see Table 1), including 45 males and 45 females of age between 18 and 22. To select the sample, a simple physical data and medical history screening, the information screen recorded by big data confirms that the test student is healthy and free of the motor system and cardiovascular and cerebrovascular diseases. The final sample size is 40 boys and 40 girls.

3.2. Experimental Research Method. To start the experimental method, firstly check the relevant data of aerobic capacity and cardiopulmonary function test experiment and collect information about young students abroad. Secondly, the test method and test equipment data process of the 20 meter turnback run are very convincing. According to the specific requirements of the national student physique, the experimental test of $1000 \mathrm{~m}$ for male students and $800 \mathrm{~m}$ for girls is conducted. Through the research of the literature, we understand that the test methods of incremental load and the differences caused by the influence of different incremental load modes clarify the monitoring indicators and evaluation significance of the maximum oxygen uptake test. This helps in placing a theoretical foundation for the demonstration and analysis of the experiment. Three kinds of aerobic capacity evaluation index test experimental methods are developed which carry out research in accordance with the experimental operating procedures.

3.2.1. Experimental Design. 80 randomly selected research subjects were divided into groups of male and female, and the experimental records of treadmill, step test, middle 
TABLE 1: Basic conditions of the aerobic test subjects (mean \pm SD).

\begin{tabular}{lcccc}
\hline Gender & Num & Age & Height $(\mathrm{cm})$ & Weight $(\mathrm{kg})$ \\
\hline Male & 40 & $20.38 \pm 1.07$ & $175 \pm 6.7$ & $64.1 \pm 5.8$ \\
Female & 40 & $20.20 \pm 0.95$ & $163.9 \pm 5.6$ & $53.6 \pm 6.1$ \\
\hline
\end{tabular}

run (male $1000 \mathrm{~m}$, girl $800 \mathrm{~m}$ ), and $20 \mathrm{~m}$ reentry running project recorded in big data were extracted. The order of the records of the experimental items extracted by the male group is treadmill, middle run, 20 m reentry run, and step test. The order in which the girl group extracts the records of the experimental items is middle run, $20 \mathrm{~m}$ reentry run, step test, and treadmill. Please note that the influence of weather factors is ignored. According to the big data records, the specific test records of each item should be at the most suitable time and weather. In addition to this, it is ensured that the interval between each two test indicators is one month for the sake of minimizing the mutual influence factors between the experimental items.

The extracted step test records should meet the requirements of the "National Student Physical Health Standards" test. Male and female subjects should be tested separately, and the step test tester (HHTL/100 physique test system) should be completed in accordance with the prescribed standard actions. 3 minutes of up and down steps should be performed, the beat is 30 times per minute. In the recovery period after the exercise load, the heart rate of $1 \sim 1.5$ minutes, 2 2.5 minutes, and 3 3.5 minutes was recorded according to the test requirements and the data required in the experimental design (with $\mathrm{HR}_{1}, \mathrm{HR}_{2}$, and $\mathrm{HR}_{3}$, respectively) representation, test indicators: $\mathrm{HR}_{1}, \mathrm{HR}_{2}, \mathrm{HR}_{3}$, PEI, and conversion equation.

$$
\text { step index }=\text { pedaling time }(s) \times \frac{100}{2 /\left(\mathrm{HR}_{1}+\mathrm{HR}_{2}+\mathrm{HR}_{3}\right)} \text {. }
$$

The equation for calculating the maximum oxygen uptake is

$$
\begin{gathered}
\text { Male } \mathrm{VO}_{2} \max \left[\frac{\mathrm{ml}}{\mathrm{min} \cdot \mathrm{kg}^{-1}}\right]=24.170+0.2588 \times \mathrm{PET}, \\
\text { Female } \mathrm{VO}_{2} \max \left[\frac{\mathrm{ml}}{\mathrm{min} \cdot \mathrm{kg}^{-1}}\right]=17.264+0.1912 \times \mathrm{PET} .
\end{gathered}
$$

The selected 20-meter test item data record should be carried out on an indoor plastic track. Before the start of the test, the subjects were tested on a $20 \mathrm{~m}$-long racetrack with end lines drawn at both ends, with the standard of stepping on or over the end line. The initial speed of the 20-MST is $8 \mathrm{~km} / \mathrm{h}$, with an increase of $0.5 \mathrm{~km} / \mathrm{h}$ every minute. If the research object cannot complete the test within the specified time for two consecutive times in the data record, the data will be eliminated. The maximum speed that the research object can accomplish is called the maximum aerobic speed (MAS). Maximum aerobic speed is calculated according to the following equation:

$$
\text { MAS }=8+0.5 \times \text { Highest level reached. }
$$

At present, the most commonly used maximum oxygen uptake conversion equation in the FITNESSGRAM test in the United States.

For age $8-18$,

$$
\begin{aligned}
\mathrm{VO}_{2} \max = & -3.238(\mathrm{MAS})-3.248(\text { Age }) \\
& +0.1536(\mathrm{MAS} \times \text { Age })+31.025 .
\end{aligned}
$$

For age 19-30,

$$
\mathrm{VO}_{2} \max =-23.4+5.8(\mathrm{MAS}) .
$$

\section{Experiments and Results}

In the "National Student Physical Fitness and Health Standard," we use the middle-distance running test item, both for male $1000 \mathrm{~m}$ and female $800 \mathrm{~m}$ to measure the cardiovascular system function in physical function and also to indirectly infer the endurance quality of adolescents. The data of the experimental subjects screened by big data select the most excellent test subjects and results. The treadmill $\mathrm{VO}_{2}$ max test method was completed in strict accordance with the plan drawn up by the laboratory test, and the test results are depicted in Figures 1 and 2. In Figure 2, the relative value and absolute value are selected having maximum oxygen uptake of male and female treadmill tests and the test results of mid-run to compare their gender differences.

4.1. Test Results. The relative and absolute values of $\mathrm{VO}_{2} \max$ for boys were $50.40 \pm 5.52 \mathrm{~mL} / \mathrm{min} \cdot \mathrm{kg}^{-1}$, and $3.19 \pm 0.27 \mathrm{~L} / \mathrm{min}$, while the relative and absolute values of $\mathrm{VO}_{2} \mathrm{max}$ for girls were $39.17 \pm 4.53 \mathrm{ml} / \mathrm{min} \cdot \mathrm{kg}^{-1}$, and $2.11 \pm 0.25 \mathrm{~L} / \mathrm{min}$, boys score $249 \pm 26.48 \mathrm{~s}$ in 1000 meters and girls score $244 \pm 20.6 \mathrm{~s}$ in 800 meters. The paired $t$-test showed that the relative value and absolute value of the maximum oxygen uptake in the treadmill test, $p$ are both less than 0.01 , showing a very significant gender difference. The middle-distance running performance showed no obvious gender difference $(p>0.05)$. Therefore, we can see that there is a certain difference in the evaluation of aerobic capacity and middle-distance running events of adolescent boys and girls through the treadmill test. The comparison of $t$ value and $p$-value of male and female treadmill tests is shown in Figure 3.

Tables 2 and 3 show that the correlation coefficient between the relative value and the absolute value of the maximum oxygen uptake of the male $1000 \mathrm{~m}$ and the treadmill test is -0.216 and -0.258 . The correlation coefficients between the relative value and absolute value of the girl's 800 meters and the maximum oxygen uptake tested on the treadmill are -0.142 and -0.223 are both negatively correlated. Related research pointed out that, $1000 \mathrm{~m}$ running is a middle-distance running, and $50 \%$ to $60 \%$ of the energy in the whole process is provided by the anaerobic system. Li Junyong et al. also found that boys' $1000 \mathrm{~m}$ performance conflicts with the relative value of $\mathrm{VO} 2$ max. 


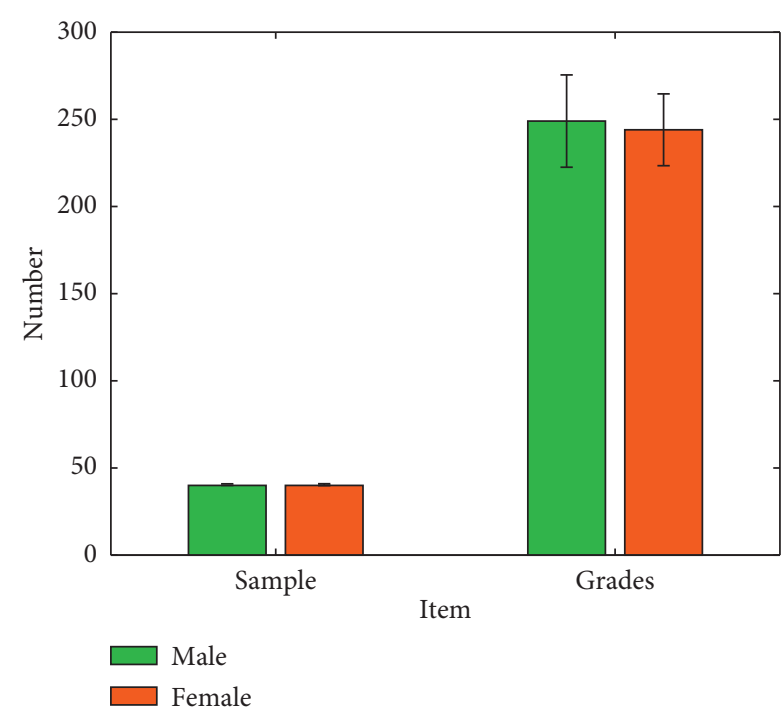

FIGURE 1: Comparison of the results of boys' 1000 meters and girls' 800 meters.

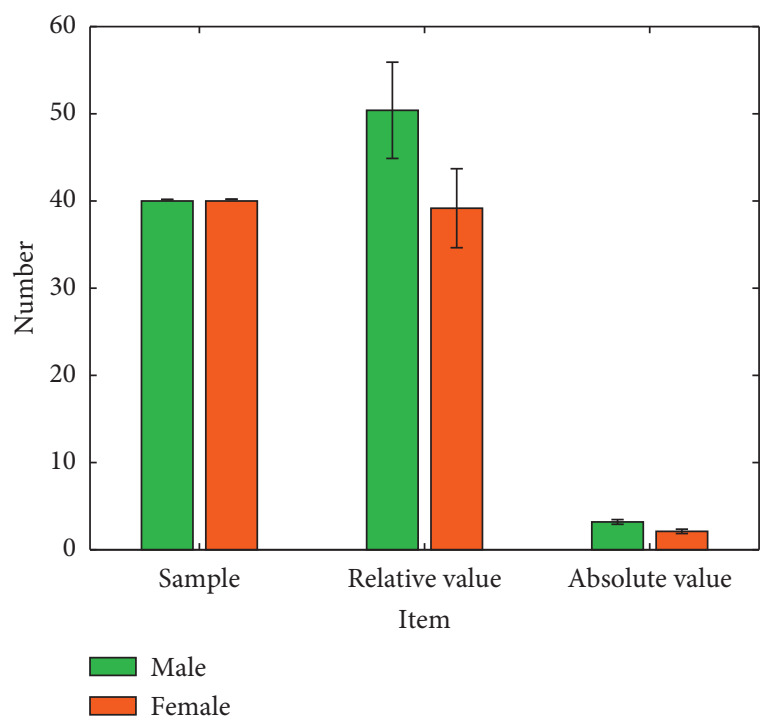

Figure 2: Relative and absolute values of maximal oxygen uptake.

Simulation results show the level of correlation between 12 minute running performance, and the absolute value of the maximum oxygen uptake is greater than the 1000-m running, which also shows that the aerobic energy supply ratio in the long-distance running of the 12-min running is greater than the $1000-\mathrm{m}$ middle-distance running. This shows that $1000 \mathrm{~m}$ running is a mixed aerobic and anaerobic exercise with anaerobic exercise as the main energy supply mode.

Therefore, it is reflected in the statistical correlation analysis that women's $800 \mathrm{~m}$ and men's $1000 \mathrm{~m}$ are negatively correlated with the relative and absolute values of the corresponding maximum oxygen uptake. Judging from the test time for the subjects to complete the $800 \mathrm{~m}$ and $1000 \mathrm{~m}$ items, the average use time for boys to complete $1000 \mathrm{~m}$ is 249 seconds, and the average use time for girls to complete $800 \mathrm{~m}$ is 244 seconds. Related foreign studies have

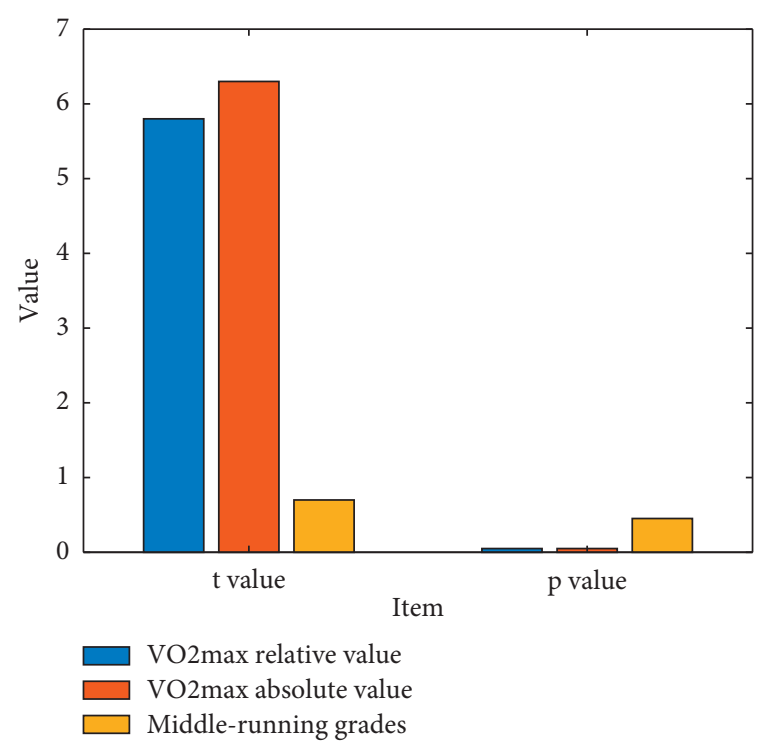

Figure 3: Comparison of $t$-value and $p$-value of male and female treadmills.

TABLE 2: Correlation between female treadmill and maximal oxygen uptake.

\begin{tabular}{lccc}
\hline Item & Treadmills $\mathrm{VO}_{2} \mathrm{max}$ & $800 \mathrm{~m}$ & $r$ \\
\hline Relative value & $39.17 \pm 4.53$ & $244.25 \pm 20.6$ & -0.142 \\
Absolute value & $2.11 \pm 0.25$ & $244.25 \pm 20.6$ & -0.223 \\
\hline
\end{tabular}

TABLE 3: Correlation between male treadmill and maximal oxygen uptake.

\begin{tabular}{lccc}
\hline Item & Treadmills $\mathrm{VO}_{2} \max$ & $800 \mathrm{~m}$ & $r$ \\
\hline Relative value & $50.40 \pm 5.52$ & $249 \pm 26.48$ & -0.216 \\
Absolute value & $3.19 \pm 0.27$ & $249 \pm 26.48$ & -0.258 \\
\hline
\end{tabular}

shown that there is a correlation between continuous running performance and maximum oxygen consumption. The effectiveness of aerobic work capacity begins to be significant after the time reaches 5 minutes under the condition of intensity maintenance exceeding $500 \%$ of the maximum oxygen uptake. It improves as the running time increases. At the same time, it is also pointed out that running at a relatively constant speed, the distance of running for 5 minutes has a moderate correlation with the maximum oxygen uptake, while the distance of running at 10 minutes shows a high correlation. According to the related reports, the total distance running in 10 minutes is highly correlated with the distance running in each unit of time during the period from 4 minutes to 10 minutes (0.81 0.99). At the same time, there is a trend that as the running distance of the subjects increases, the closer the total distance from the last 10 minutes, the higher the correlation. For the relevant research between different running distances and $\mathrm{VO}_{2}$ max, it is pointed out that below $400 \mathrm{~m}$ is the low correlation with $\mathrm{VO}_{2} \max$, and long-distance running or $12 \mathrm{~min}$ running over $3000 \mathrm{~m}$ has the highest correlation with $\mathrm{VO}_{2} \max$. 
Therefore, we can see that the middle-distance race (male $1000 \mathrm{~m} /$ female $800 \mathrm{~m}$ ) has a low correlation with the maximum oxygen uptake in both the running time and the running distance, which is also consistent with the results in this study. In addition, as a test item for the standard assessment, young students will change their running rhythm, speed, physical fitness, and psychology in order to pass the assessment. This may also lead to a negative correlation between the measured value of the mid-run test and the measured maximum oxygen uptake value.

\section{Conclusion}

Facing the outstanding problems in the physical health of adolescents in the new era, the research on the evaluation of the physical health of adolescents around the world has focused on the core content of health quality. In China, currently "National Student Physical Health Standards" has retained a lot of evaluation content related to sports technical quality. In view of the current development trend of youth physical health evaluation and the exchange and comparison with international youth physical health, the physical four areas of composition, aerobic capacity, muscle strength and muscular endurance, and joint flexibility, are defined, and the indicators related to health are selected for testing and evaluation, which objectively and accurately reflect the physical health of young students. The results of the correlation experiment analysis on the aerobic capacity evaluation indicators show that the test value of the middle running (male $1000 \mathrm{~m}$, female $800 \mathrm{~m}$ ) currently used in China is negatively correlated with the relative value of the measured maximum oxygen uptake on the treadmill (male $r=-0.216$, female $r=-0.142$ ); the result analysis shows that, in the middle running project, anaerobic exercise is the main energy supply mode. In aerobic and anaerobic mixed exercise, more than half of the energy is provided by the anaerobic system. The correlation between the estimated value of the step test and the measured value of the treadmill shows that male $r=0.497, p<0.01$; girl $r=0.413$, $p \leq 0.05$, which is significant, but from the fitting coefficient. Considering, male $L=0.267$, female $L=0.188$, the fit is low; there is no linear relationship, and it cannot accurately evaluate the aerobic capacity of adolescents. The correlation between the estimated value of the $20 \mathrm{~m}$ round-trip run and the measured value of the treadmill shows male $r=0.796, p<0.01$, female $r=0.748, p<0.01$; there is a very significant correlation and from in terms of fitting coefficient, male $L=0.634$, female $L=0.599$, the fitting coefficient is high, and the linear relationship is obvious. The experimental results show that it is more appropriate to select the $20 \mathrm{~m}$ reentry run as a test index to evaluate the aerobic capacity of adolescents.

\section{Data Availability}

The data used to support the findings of this study are available from the corresponding author upon request.

\section{Conflicts of Interest}

The author declares that he has no conflicts of interest.

\section{References}

[1] Y. Li, "Comparative analysis of BMI index method and the "national student health standards"," Sports Forum, vol. 4, 2011.

[2] Z. P. Zhen and Z. M. Mao, "Research on the structure and change of national student's physical fitness and health standards index system," Journal of Xi'an Physical Education University, vol. 4, 2008.

[3] R. R. Pate, S. G. Trost, S. Levin, and M. Dowda, "Sports participation and health-related behaviors among US youth," Archives of Pediatrics and Adolescent Medicine, vol. 154, no. 9, pp. 904-911, 2000.

[4] H. Howald, "Sport und Gesundheit," Sozial-und Präventivmedizin SPM, vol. 21, no. 6, pp. 241-244, 1976.

[5] G. Tanaka, "Method of executing benchmark test," US Patent, 2002.

[6] W. Yan, "Analysis of "national student physical health standard" content adjustment," Wushu Science, vol. 5, 2014.

[7] R. Wang and Z. P. Wang, "Interpretation and analysis of trial program of standards for students' physical constitution and health," Journal of Physical Education, vol. 5, 2004.

[8] R. Bush Nicole, R. D. Lane, and K. A. McLaughlin, "Introduction to the special issue of psychosomatic medicine: mechanisms linking early-life adversity to physical health," Psychosomatic Medicine Journal of the American Psychosomatic Society, vol. 78, no. 9, 2016.

[9] Y. Pin-Liang, "The experiment research on the effect of aerobics exercise to the polytechnic girl's body shape, sport capability and physiological function," Fujian Sports Science and Technology, vol. 6, 2003.

[10] R. S. Paffenbarger Jr., J. N. Morris, W. L. Haskell, P. D. Thompson, and I.-M. Lee, "An introduction to the journal of physical activity and health," Journal of Physical Activity and Health, vol. 1, no. 1, 2004.

[11] C. Zhang, "Review on models of mental health education in middle schools and elementary schools at home and abroad," Chinese Journal of Special Education, vol. 9, 2006.

[12] W. A. Wallis, "At home and abroad," Society, vol. 22, no. 1, pp. 7-10, 1984.

[13] C. Yunwen, F. Lichun, and W. Liqing, "A comparison of health education about neonatal jaundice at home and abroad," The Medical Forum, vol. 4, 2018.

[14] J. Cirne, R. Dormeval, and E. Al, "A practical constitutive model covering a wide range of strain rates and a large region of strain," Journal de Physique IV, vol. 134, pp. 55-61, 2006.

[15] K. E. Zunyu, "The reform of physical fitness measurement in Japan,” China Sport Science and Technology, vol. 5, 2003.

[16] S. L. Manxi Zheng, "The research of college students' physique health and relevant interventions," Journal of Inner Mongolia Normal University, vol. 6, 2013.

[17] J. L. Zhao, S. Y. Zhang, and M. A. Zhen-Quan, "Influence of physical interventions on physiques and health of college students," Journal of Lianyungang Technical College, vol. 11, 2010.

[18] X. Liu, "A study on the theory and practice teaching in sports measurement and evaluation," Journal of Wuhan Institute of Physical Education, vol. 8, 1998.

[19] B. Kegel, "Sports and recreation for those with lower limb amputation or impairment," Journal of Rehabilitation Research and Development, vol. 1, no. 1, pp. 1-125, 1985.

[20] D. A. Brodie and K. Stopani, "Experimental ethics in sports medicine research," Sports Medicine, vol. 9, no. 3, pp. 143-150, 1990. 